\title{
Evaluation of Vesical Imaging-Reporting and Data System (VI-RADS) scoring system in predicting muscle invasion of bladder cancer
}

\author{
Shenghua Liu ${ }^{1 \#}$, Feijia $\mathrm{Xu}^{2 \#}$, Tianyuan $\mathrm{Xu}^{1}$, Yang Yan ${ }^{1}$, Xudong Yao ${ }^{1}$, Guangyu Tang ${ }^{2}$ \\ ${ }^{1}$ Department of Urology, ${ }^{2}$ Department of Radiology, Shanghai Tenth People's Hospital, Tongji University, Shanghai 200072, China \\ Contributions: (I) Conception and design: S Liu; (II) Administrative support: X Yao, G Tang; (III) Provision of study materials or patients: F Xu; (IV) \\ Collection and assembly of data: S Liu, T Xu; (V) Data analysis and interpretation: S Liu, F Xu; (VI) Manuscript writing: All authors; (VII) Final \\ approval of manuscript: All authors. \\ \#These authors contributed equally to this work. \\ Correspondence to: Xudong Yao; Guangyu Tang. No. 301 Mid Yanchang Road, Shanghai 200072, China. Email: yaoxudong1967@163.com; \\ drtangguangyu@126.com.
}

Background: To investigate the accuracy of using the Vesical Imaging-Reporting and Data System (VIRADS) scoring system in prediction preoperative muscle invasion of bladder cancer.

Methods: The study retrospectively reviewed consecutive patients with bladder cancer who received multiparametric magnetic resonance imaging (MRI) between January 2017 and June 2019. Clinical and pathological parameters were collected. Bladder tumors were re-evaluated with 5-point VI-RADS scoring system by two experienced radiologists independently. The VI-RADS score was compared with postoperative pathology for each tumor for determining muscle invasion. Sensitivity, specificity, positive predictive value (PPV), and negative predictive value (NPV) were calculated for each VI-RADS cutoff.

Results: A total of 126 patients were included in analysis, with 82 patients received transurethral resection of bladder tumor (TURBt) while 44 underwent radical cystectomy. Fifty patients were muscle-invasive bladder cancer and 76 were non-muscle invasive tumor confirmed pathologically. VI-RADS score was only predictive factor to muscle invasion in multivariate analysis. Setting VI-RADS score greater than or equal to 4 reached the best sensitivity and specificity of $94.00 \%$ and $92.11 \%$, with PPV and NPV value of $88.68 \%$ and $95.89 \%$.

Conclusions: VI-RADS score system is a promising and effective modality in determining detrusor muscle invasion of bladder cancer preoperatively.

Keywords: Bladder cancer; Vesical Imaging-Reporting and Data System (VI-RADS); multiparametric magnetic resonance imaging (mpMRI); muscle invasion; prediction

Submitted Sep 28, 2019. Accepted for publication Feb 14, 2020.

doi: $10.21037 /$ tau.2020.02.16

View this article at: http://dx.doi.org/10.21037/tau.2020.02.16

\section{Introduction}

Bladder cancer is the most common cancer in the urinary system with high morbidity and relapse rate. It is the fourth common cancer in men and the seventh the most common malignancy in the world. It is estimated that 80,470 new cases and 17,670 deaths from bladder cancer will occur in the United States in $2019(1,2)$. Non-muscle invasive bladder cancer could be treated by transurethral resection (TUR) while muscle invasive bladder cancer must receive radical cystectomy as golden standard $(3,4)$. Therefore, preoperative accurate staging is vital to ensure correct treatment choice and prognosis prediction.

Magnetic resonance imaging (MRI) has long been recognized as one of the best imaging modalities for 
its high spatial and contrast resolution. The state of art multiparametric MRI (mpMRI), a combination of T2weighted, diffusion-weighted and dynamic gadoliniumenhanced imaging sequences provided value in detection, localization and staging in bladder cancer patients (5). Several meta-analyses showed MRI had a good diagnostic performance for prediction muscle invasion of bladder cancer $(6,7)$. However, previous studies lack uniform MRI evaluation criteria and therefore limit its promotion as an excellent staging method for bladder cancer.

In the year 2018, the Vesical Imaging-Reporting and Data System (VI-RADS) was released as a standard imaging protocol and reporting criteria (including size, location, multiplicity, and morphology) for bladder mpMRI (8). The novel scoring system classifies the possibility of muscle invasion into five stages, which could provide a more meticulous distinction between clinical T1 and T2 stage lesions that used to be hard to distinguish by conventional MRI interpretation. Before it could be widely accepted and adopted, its clinical validation should be tested. In our study, we aimed to retrospectively evaluate the accuracy by using VI-RADS to discriminate the muscle invasion in bladder cancer patients.

\section{Methods}

\section{Patient selection and data collection}

We retrospectively reviewed the medical information of consecutive patients who were diagnosed as bladder cancer in Shanghai Tenth People's Hospital between January 2017 to June 2019. Clinicopathological information including age, gender, tumor stage, grade was collected for those who received mpMRI before surgery, either transurethral resection of bladder tumor (TURBt) or radical cystectomy. After surgery the resected tissue or organ was sent for pathological evaluation routinely. For TURBt, a standard resection of tumor was performed by two experienced hands (X Yao and Y Yan) with the depth to at least detrusor muscle layer. The including criteria was (I) mpMRI was performed within 2 weeks before surgery; (II) the pathology was urothelial carcinoma. Patients with treatment prior to mpMRI, such as TURBt within 3 months, biopsy within 2 weeks or cystoscopy within 3 days were excluded due to possible judgment interference. The study was approved by the institutional review board of Shanghai Tenth People's hospital (No. 2019-321).

\section{MRI Protocol}

All exams were performed using a 3-T MRI scanner (Magnetom Verio: Siemens, Erlangen, Germany), equipped with an 8-channel phased-array coil. Patients were instructed to start drinking $500-1,000 \mathrm{~mL}$ of water in 30 min before scanning to obtain an optimally full bladder (around $300 \mathrm{~mL}$ ). The scanning protocol of the pelvis include the whole bladder, proximal urethra, pelvic nodes and prostate if the patient is a male. If the patient is a female, uterus, ovaries, fallopian tubes, and vagina need to be included.

Axial T1-weighted [repetition time/echo time (TR/TE), 600/11], turbo-spin-echo nonfat-suppressed T2-weighted in axial and coronal planes and turbo-spin-echo fat-suppressed T2-weighted in sagittal plane were performed.

Diffusion-weighted imaging (DWI) were performed with breathing-free spin-echo echo planar imaging (EPI) sequence in axial including high b value $\left(800-1,000 \mathrm{~s} / \mathrm{mm}^{2}\right)$ to display bladder cancer with high contrast to surrounding tissues;

Axial dynamic contrast-enhanced imaging (DCE) was performed after injection of gadopentetate administered at a dose of $0.1 \mathrm{mmol} / \mathrm{kg}$ at a rate of $1.5-2.0 \mathrm{~mL} / \mathrm{s}$. A $5-6$ sets of CE images including three orthogonal planes were acquired 20-131 seconds after injection of contrast agents. Pre-contrast imaging was also needed.

Bladder MR imaging protocol parameters are summarized in Table 1.

\section{MRI interpretation}

MRI images were independently re-evaluated by two experienced radiologists ( $\mathrm{F} \mathrm{Xu}$ and $\mathrm{T} \mathrm{Xu}$ ), ignorant of the patients' medical history and pathological results, on the basis of 5-point VI-RADS scoring system. They have studied the VI-RADS algorithm for at least 1 week before practicing. Number of lesions, tumor size and their locations were recorded according to the schematic map (8). Hydronephrosis was defined as dilatation of the ureter due to bladder cancer obstruction at the time of surgery. When multiple lesions were presented, tumor size were measured as the diameter of the largest lesion, and VI-RADS score were considered as the highest one. When discordance was seen between two interpreters, consultation was conducted to reach consensus. 
Table 1 MRI parameter setting

\begin{tabular}{lcccccc}
\hline Parameters & TR $(\mathrm{ms})$ & TE $(\mathrm{ms})$ & Flip angle (degree) & FOV $(\mathrm{cm})$ & Slice thickness $(\mathrm{mm})$ & B value \\
\hline T2Wl cor & 5,000 & 120 & 120 & 22 & 4 & - \\
T2Wl sag & 4,000 & 85 & 140 & 30 & 5 & - \\
T2WI tra & 4,050 & 85 & 150 & 90 & 26 & $6,50,800,1,000$ \\
DWI & 4,500 & 72 & 9 & 26 & - \\
DCE & 3.9 & 1.4 & 9 & - \\
\hline
\end{tabular}

MRI, magnetic resonance imaging; TR, repetition time; TE, echo time; FOV, field of view; T2WI, T2 weighted image; cor, coronal; sag, sagittal; tra, tranverse.

\section{Statistical analysis}

The cohort characteristics were described based on different VI-RADS score groups. Clinicopathological variables were compared dichotomized according to the muscle invasion status (stage T2 or higher). The Student $t$-test, Wilcoxon signed rank test, and Chi-square test were used to estimate differences of clinical and pathological characteristics between groups. Multivariate logistic regression model was performed to identify predictive factors of a muscle invasive tumor, using the variables statistically significant in univariate analysis. All statistical tests were two-tailed, with $\mathrm{P}<0.05$ considered significant. Sensitivity, specificity, positive predictive value (PPV), and negative predictive $(\mathrm{NPV})$ value were calculated using a $2 \times 2$ contingency table, and for each VI-RADS category used as cutoff. Statistical analyses were performed with SPSS 20.0 (SPSS Inc., Chicago, IL, USA).

\section{Results}

A total of 126 patients, with the mean age of 68 years old were enrolled in the study, including 104 males and 22 females. Eighty-two of them received TURBt while 44 underwent radical cystectomy. The number of patients with VI-RADS score of 1 to 5 were 16, 22, 35, 18 and 35 respectively. The characteristic of the cohort was listed in Table 2. Of all the patients, 76 were non-muscle invasive and the rest 50 were muscle invasive tumor. Patients with muscle invasive tumor had features of preoperative hydronephrosis, larger tumor size $(\geq 3 \mathrm{~cm})$, multifocality and higher VI-RADS score (all $\mathrm{P}<0.05$ ) (Table 3). To figure out which factor remain the most vital in differentiating muscle invasive tumor, multivariate logistic analysis was performed and the result showed that only VI-RADS score were statistically significant $(\mathrm{P}<0.001)$ (Table 4).

The ability of VI-RADS score to define the muscle invasive tumor were calculated according to different cutoff value. The sensitivity and specificity were $100.00 \%$ and $21.05 \%$ when the cut-off VI-RADS $\geq 2$ was used. When the cut-off moves to VI-RADS $\geq 3$, the sensitivity and specificity came to $100.00 \%$ and $50.00 \%$, respectively. However, if VI-RADS score was set as $\geq 4$, the sensitivity and specificity could be $94.00 \%$ and $92.11 \%$, with PPV and NPV value were $88.68 \%$ and $95.89 \%$. If the cut-off was set as VIRADS $\geq 5$, sensitivity fell to only $68.00 \%$ (Table 5 ).

The receiver operating characteristic (ROC) curve showed comparing with other parameters, VI-RADS score had the best ability to discriminate muscle invasive tumor (Figure 1). The area under curve (AUC) value was 0.966 (95\% CI, 0.937 to 0.996 ) for VI-RADS, 0.681 (95\% CI 0.580 to 0.781 ) for hydronephrosis, 0.879 ( $95 \%$ CI, 0.810 to 0.937 ) for tumor size, 0.392 (95\% CI, 0.293 to 0.491 ) for number of lesions, respectively.

\section{Discussion}

In our retrospective study, we assessed the ability of mpMRI for detection of muscle invasion bladder cancer based on the newly released 5-point VI-RADS score. Overall, mpMPI showed a good performance with the accuracy of $92.8 \%$ when setting the cut-off value as VI-RADS 3.

To judge the muscle invasion would be crucial to decide the treatment strategy. Although several molecular panels have been figured out to predict muscle invasion in high risk non-muscle invasive tumor $(9,10)$, the best of art currently was to perform TURBt, waiting for the pathological outcomes. However, the significant risk of residual tumor after primary TUR has been demonstrated (11). A systematic review demonstrated a $51 \%$ risk of disease 
Table 2 Characteristic of patients categorized to different VI-RADS score

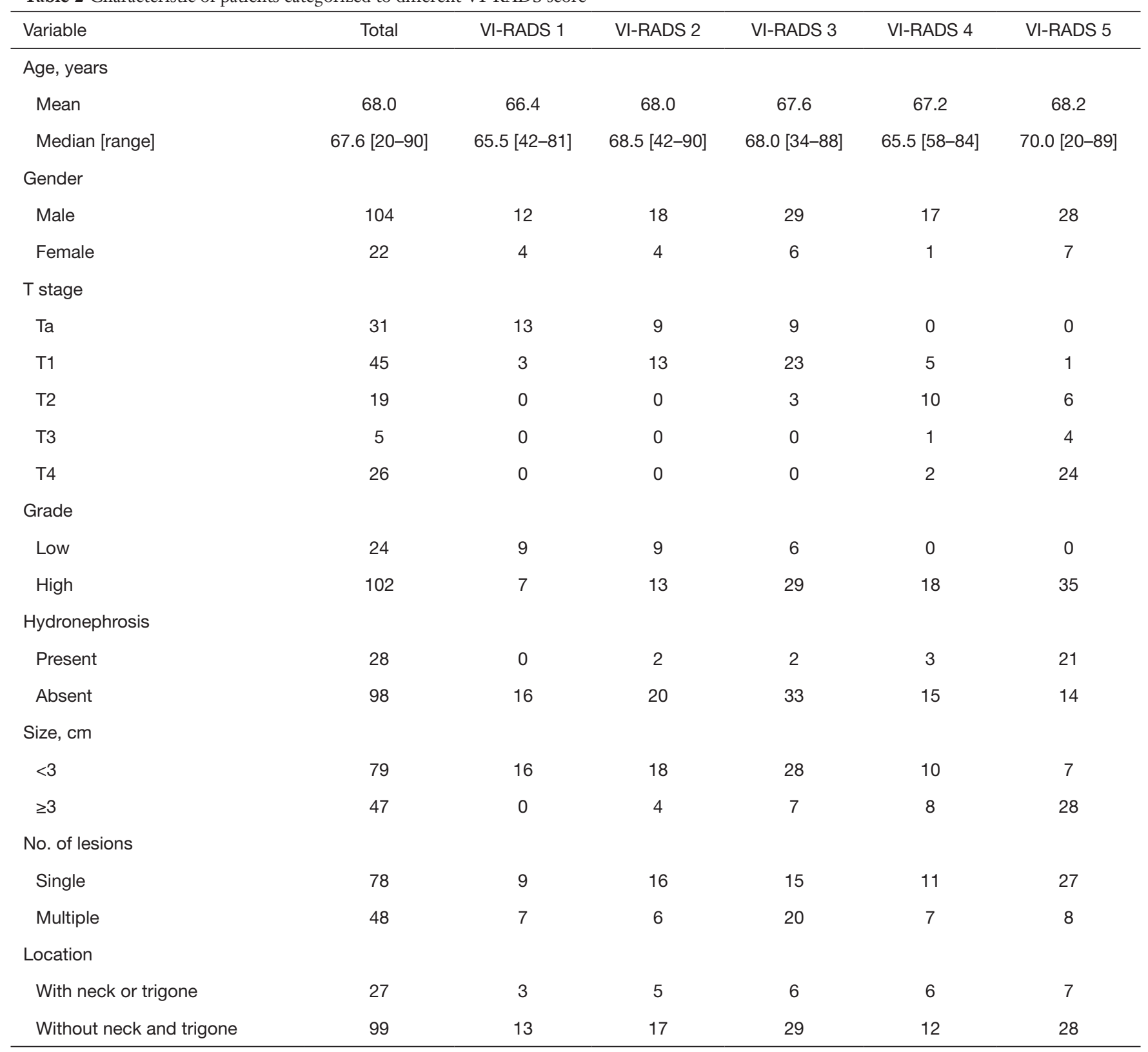

VI-RADS, Vesical Imaging-Reporting and Data System.

persistence and an $8 \%$ risk of understaging in T1 tumors. Most of the residual lesions were detected at the original tumor location (12). This indicated mere TURBt cannot fully reflected the tumor de facto stage. Therefore, if preoperative imaging modality such as mpMRI could provide reliable staging information, the surgeon could be more confident in performing a curative TURBT.

Researches in the past decade have showed MRI as a useful method for the localization and assessment of bladder tumor extension and adjacent organ involvement. Dynamic contrast enhancing MRI are valuable indicators for assessing tumor angiogenic activity and tumor neovascularization (13). Diffusion-weighted MRI might have high specificity for the detection of suspected bladder cancer and invasion (14). To promote further generalization of $\mathrm{mpMRI}$ into research and clinical practice, an expert group recently issued a 5-point 
Table 3 Characteristics of patients categorized to muscle invasiveness

\begin{tabular}{|c|c|c|c|}
\hline Variables & $\begin{array}{c}\text { Non-muscle } \\
\text { invasive }\end{array}$ & $\begin{array}{l}\text { Muscle- } \\
\text { invasive }\end{array}$ & $P$ value \\
\hline Gender & & & 0.726 \\
\hline Male & 62 & 42 & \\
\hline Female & 14 & 8 & \\
\hline Hydronephrosis & & $<0.001$ & \\
\hline Absent & 70 & 28 & \\
\hline Present & 6 & 22 & \\
\hline Location & & & 0.534 \\
\hline With neck or trigone & 16 & 11 & \\
\hline Without neck and trigone & 60 & 39 & \\
\hline Size, cm & & & $<0.001$ \\
\hline$<3$ & 64 & 15 & \\
\hline$\geq 3$ & 12 & 35 & \\
\hline No. of lesions & & 0.009 & \\
\hline Single & 40 & 38 & \\
\hline Multiple & 36 & 12 & \\
\hline VI-RADS & & & $<0.001$ \\
\hline 1 & 16 & 0 & \\
\hline 2 & 22 & 0 & \\
\hline 3 & 32 & 3 & \\
\hline 4 & 5 & 13 & \\
\hline 5 & 1 & 34 & \\
\hline
\end{tabular}

VI-RADS, Vesical Imaging-Reporting and Data System.

scoring system in order to standardize its utility (8). The VI-RADS scoring system, however, is still in its early stage, and therefore demands further validation.

Our results showed that all lesions scored as VI-RADS 1 and 2 were verified as non-muscle invasive. For VIRADS 3 lesions, 32 out of 35 were non-muscle invasive. When it comes to VI-RADS 4 and 5, the percentage of muscle invasive tumor were as high as $72.2 \%$ and $97.1 \%$, respectively. Thus, when setting VI-RADS score greater than 3 as cutting off value, both the sensitivity and specificity could reach the best. To our knowledge, only several similar studies had investigated the discrimination ability of VI-RADS scoring system on muscle invasion,
Table 4 Multivariate logistic regression model evaluating the risk factor of muscle invasiveness

\begin{tabular}{lccc}
\hline Variable & HR & $95 \% \mathrm{Cl}$ & P value \\
\hline VI-RADS & 25.090 & $6.969-90.334$ & $<0.001$ \\
Hydronephrosis & 0.343 & $0.036-3.241$ & 0.350 \\
Size & 2.176 & $0.450-10.521$ & 0.334 \\
No. of lesions & 0.261 & $0.054-1.274$ & 0.097 \\
\hline
\end{tabular}

VI-RADS, Vesical Imaging-Reporting and Data System; HR, hazard ratio; $\mathrm{Cl}$, confidence interval.

Table 5 Sensitivity, specificity, positive predict value and negative predict value for each cutoff

\begin{tabular}{lcccc}
\hline Variables & $\begin{array}{c}\text { Sensitivity } \\
(\%)\end{array}$ & $\begin{array}{c}\text { Specificity } \\
(\%)\end{array}$ & $\begin{array}{c}\text { Positive } \\
\text { predict } \\
\text { value (\%) }\end{array}$ & $\begin{array}{c}\text { Negative } \\
\text { predict } \\
\text { value (\%) }\end{array}$ \\
\hline VI-RADS $\geq 2$ & 100.00 & 21.05 & 45.45 & 100.00 \\
VI-RADS $\geq 3$ & 100.00 & 50.00 & 56.82 & 100.00 \\
VI-RADS $\geq 4$ & 94.00 & 92.11 & 88.68 & 95.89 \\
VI-RADS $\geq 5$ & 68.00 & 98.68 & 97.14 & 82.42 \\
\hline VI-RADS, Vesical Imaging-Reporting and Data System.
\end{tabular}

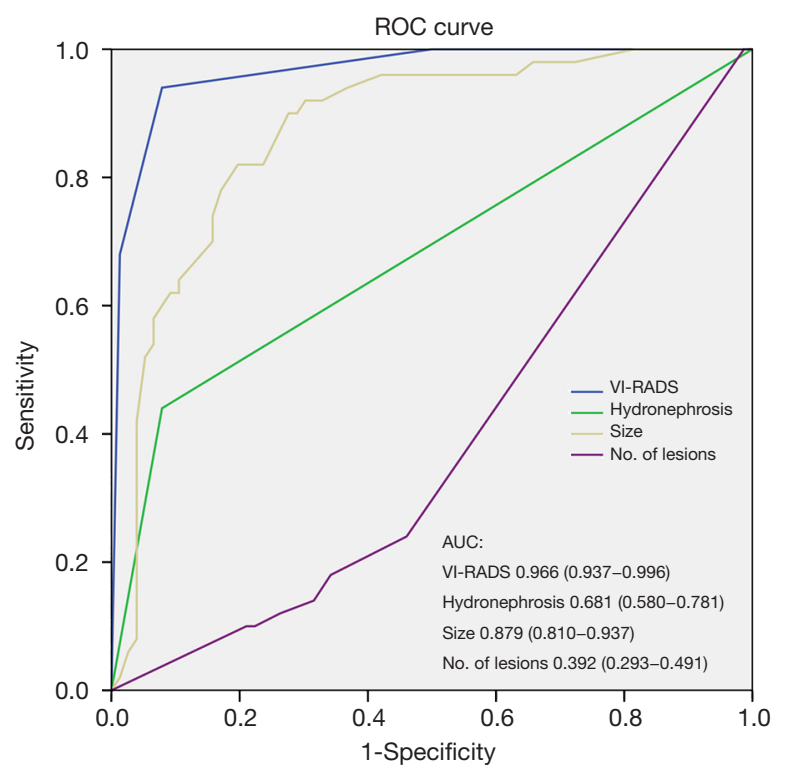

Figure 1 Receiver operating characteristic (ROC) curve of different variables for predicting muscle-invasive bladder cancer. The area under curve and $95 \%$ confidence interval were showed in the lower right part (All $\mathrm{P}<0.05)$. AUC, area under the curve; VIRADS, Vesical Imaging-Reporting and Data System. 
most of which are small samples $(15,16)$. Barchetti et al. including 78 patients showed VI-RADS score great than 3 had sensitivity of $77-82 \%$ and specificity $89-94 \%$ (16). Another largest study hitherto retrospectively reviewed 340 patients found that setting VI-RADS score great than 2 had best sensitivity and specificity of $87.1 \%$ and $96.5 \%$ respectively. For tumors scored as VI-RADS 3, 33.3\% were confirmed to be non-muscle invasive cancer and $66.7 \%$ were confirmed to be muscle-invasive cancer (17). These indicate there is great uncertainty about the proportion of muscle invasion among VI-RADS 3 patients. Would VIRADS score of 3 be a gray zone in defining the depth of tumor invasion warrant further researches.

Our study also analysis several preoperative factors supposed to be linked with muscle invasion, such as number of lesions, tumor size and hydronephrosis. Preoperative hydronephrosis were associated with advanced tumor stage and lymph node invasion (18). Tumor size great than or equal to $3 \mathrm{~cm}$, multifocality were correlated with recurrence and progression for non-muscle invasive tumor to muscle invasion $(19,20)$. Bladder neck and/or trigone involving were also predictive factors for progression and worse survival, possibly because of the proximity to vascular bed and thinness of lamina proper (21-23). However, together with VI-RADS score, these above factors were not statistically significant under multivariate analysis, indicating that preoperative mpMRI evaluation outweighed all the clinical variables in distinguishing muscle invasion.

Our study has limitations. First, it is a single-center, retrospective research with unavoidable selection bias. Patients who received mpMRI before operation were likely to bear heavier tumor burden and intended to receive radical cystectomy. Second, the image evaluation was performed by radiologists with expertise on urology. This could make its application in grass-roots hospital difficult for lack of experience. Third, 65\% of pathology were evaluated by TURBt as golden standard, which might cause staging inaccuracy. Upstaging might occur in nearly onethird of patients who were initial Ta or T1 stage on TURBt after radical cystectomy (24). Whatever, our study provided one of the few evidences verifying the usefulness of VIRADS score system.

\section{Conclusions}

To summarize, using VI-RADS score system in mpMRI could be a promising and effective method in differentiating muscle invasion of bladder cancer. Whether tumor of VI-
RADS 3 invade detrusor muscle should be judge with caution. Therefore, further multi-centered, large research of its application is warrant.

\section{Acknowledgments}

Funding: The study was supported by the Fundamental Research Funds for the Central Universities (No. 22120180580).

\section{Footnote}

Conflicts of Interest: All authors have completed the ICMJE uniform disclosure form (available at http://dx.doi. org/10.21037/tau.2020.02.16). The authors have no conflicts of interest to declare.

Ethical Statement: The authors are accountable for all aspects of the work in ensuring that questions related to the accuracy or integrity of any part of the work are appropriately investigated and resolved. The study was approved by the institutional review board of Shanghai Tenth People's hospital (No. 2019-321).

Open Access Statement: This is an Open Access article distributed in accordance with the Creative Commons Attribution-NonCommercial-NoDerivs 4.0 International License (CC BY-NC-ND 4.0), which permits the noncommercial replication and distribution of the article with the strict proviso that no changes or edits are made and the original work is properly cited (including links to both the formal publication through the relevant DOI and the license). See: https://creativecommons.org/licenses/by-nc$\mathrm{nd} / 4.0 /$.

\section{References}

1. Siegel RL, Miller KD, Jemal A. Cancer statistics, 2018. CA Cancer J Clin 2018;68:7-30.

2. Ploeg M, Aben KK, Kiemeney LA. The present and future burden of urinary bladder cancer in the world. World J Urol 2009;27:289-93.

3. Babjuk M, Bohle A, Burger M, et al. EAU Guidelines on Non-Muscle-invasive Urothelial Carcinoma of the Bladder: Update 2016. Eur Urol 2017;71:447-61.

4. Alfred Witjes J, Lebret T, Comperat EM, et al. Updated 2016 EAU Guidelines on Muscle-invasive and Metastatic Bladder Cancer. Eur Urol 2017;71:462-75. 
5. van der Pol CB, Chung A, Lim C, et al. Update on multiparametric MRI of urinary bladder cancer. J Magn Reson Imaging 2018;48:882-96.

6. Woo S, Suh CH, Kim SY, et al. Diagnostic performance of MRI for prediction of muscle-invasiveness of bladder cancer: A systematic review and meta-analysis. Eur J Radiol 2017;95:46-55.

7. Gandhi N, Krishna S, Booth CM, et al. Diagnostic accuracy of magnetic resonance imaging for tumour staging of bladder cancer: systematic review and metaanalysis. BJU Int 2018;122:744-53.

8. Panebianco V, Narumi Y, Altun E, et al. Multiparametric Magnetic Resonance Imaging for Bladder Cancer: Development of VI-RADS (Vesical Imaging-Reporting And Data System). Eur Urol 2018;74:294-306.

9. D Andrea D, Abufaraj M, Susani M, et al. Accurate prediction of progression to muscle-invasive disease in patients with pT1G3 bladder cancer: A clinical decisionmaking tool. Urol Oncol 2018;36:239.e1-239.e7.

10. Kang HW, Seo SP, Byun YJ, et al. Molecular Progression Risk Score for Prediction of Muscle Invasion in Primary T1 High-Grade Bladder Cancer. Clin Genitourin Cancer 2018;16:274-80.

11. Brausi M, Collette L, Kurth K, et al. Variability in the recurrence rate at first follow-up cystoscopy after TUR in stage Ta $\mathrm{T} 1$ transitional cell carcinoma of the bladder: a combined analysis of seven EORTC studies. Eur Urol 2002;41:523-31.

12. Cumberbatch MGK, Foerster B, Catto JWF, et al. Repeat Transurethral Resection in Non-muscle-invasive Bladder Cancer: A Systematic Review. Eur Urol 2018;73:925-33.

13. Hassanien OA, Abouelkheir RT, Abou El-Ghar MI, et al. Dynamic Contrast-Enhanced Magnetic Resonance Imaging as a Diagnostic Tool in the Assessment of Tumour Angiogenesis in Urinary Bladder Cancer. Can Assoc Radiol J 2019;70:254-63.

14. Halefoglu AM, Sen EY, Tanriverdi O, et al. Utility of diffusion-weighted MRI in the diagnosis of bladder carcinoma. Clin Imaging 2013;37:1077-83.

Cite this article as: Liu S, Xu F, Xu T, Yan Y, Yao X, Tang G. Evaluation of Vesical Imaging-Reporting and Data System (VIRADS) scoring system in predicting muscle invasion of bladder cancer. Transl Androl Urol 2020;9(2):445-451. doi: 10.21037/ tau.2020.02.16
15. Makboul M, Farghaly S, Abdelkawi IF. Multiparametric MRI in differentiation between muscle invasive and non-muscle invasive urinary bladder cancer with vesical imaging reporting and data system (VI-RADS) application. Br J Radiol 2019;92:20190401.

16. Barchetti G, Simone G, Ceravolo I, et al. Multiparametric MRI of the bladder: inter-observer agreement and accuracy with the Vesical Imaging-Reporting and Data System (VI-RADS) at a single reference center. Eur Radiol 2019;29:5498-506.

17. Wang H, Luo C, Zhang F, et al. Multiparametric MRI for Bladder Cancer: Validation of VI-RADS for the Detection of Detrusor Muscle Invasion. Radiology 2019;291:668-74.

18. Oh JJ, Byun SS, Jeong CW, et al. Association Between Preoperative Hydronephrosis and Prognosis After Radical Cystectomy Among Patients With Bladder Cancer: A Systemic Review and Meta-Analysis. Front Oncol 2019;9:158.

19. Zachos I, Tzortzis V, Mitrakas L, et al. Tumor size and $\mathrm{T}$ stage correlate independently with recurrence and progression in high-risk non-muscle-invasive bladder cancer patients treated with adjuvant BCG. Tumour Biol 2014;35:4185-9.

20. Rodríguez Faba O, Palou J. Predictive factors for recurrence progression and cancer specific survival in high-risk bladder cancer. Curr Opin Urol 2012;22:415-20.

21. Miyazaki J, Nishiyama H. Epidemiology of urothelial carcinoma. Int J Urol 2017;24:730-4.

22. Fujii Y. Prediction models for progression of nonmuscle-invasive bladder cancer: A review. Int J Urol 2018;25:212-8

23. Kobayashi S, Fujii Y, Koga F, et al. Impact of bladder neck involvement on progression in patients with primary nonmuscle invasive bladder cancer: a prospective validation study. Urol Oncol 2014;32:38.e29-36.

24. Turker P, Bostrom PJ, Wroclawski ML, et al. Upstaging of urothelial cancer at the time of radical cystectomy: factors associated with upstaging and its effect on outcome. BJU Int 2012;110:804-11. 